\title{
Nonlinear transport phenomena in a two-subband system
}

\author{
S. Wiedmann, ${ }^{1,2,3}$ G. M. Gusev, ${ }^{4}$ O. E. Raichev,${ }^{5}$ A. K. Bakarov,${ }^{6}$ and J. C. Portal ${ }^{2,3}$ \\ ${ }^{1}$ High Field Magnet Laboratory, Institute for Molecules and Materials, Radboud University Nijmegen, \\ Toernooiveld 7, 6525 ED Nijmegen, the Netherlands \\ ${ }^{2}$ Laboratoire National des Champs Magnétiques Intenses, CNRS-UJF-UPS-INSA, 38042 Grenoble, France \\ ${ }^{3}$ INSA Toulouse, 31077 Toulouse Cedex 4, France \\ ${ }^{4}$ Instituto de Física da Universidade de São Paulo, Caixa Postale 66318, São Paulo, São Paulo, Brazil \\ ${ }^{5}$ Institute of Semiconductor Physics, NAS of Ukraine, Prospekt Nauki 41, 03028, Kiev, Ukraine \\ ${ }^{6}$ Institute of Semiconductor Physics, Novosibirsk 630090, Russia
}

(Received 24 June 2011; published 4 October 2011)

\begin{abstract}
We study nonlinear transport phenomena in a high-mobility bilayer system with two closely spaced populated electronic subbands in a perpendicular magnetic field. For a moderate direct current excitation, we observe zero-differential-resistance states with a characteristic $1 / B$ periodicity. We investigate, both experimentally and theoretically, the Hall-induced resistance oscillations which modulate the high-frequency magnetointersubband oscillations in our system if we increase the current. We also observe and describe the influence of direct current on the magnetoresistance in the presence of microwave irradiation.
\end{abstract}

DOI: 10.1103/PhysRevB.84.165303

PACS number(s): 73.40.-c, 73.43.-f, 73.21.-b

\section{INTRODUCTION}

Nonequilibrium magnetotransport in two-dimensional electron systems (2DESs) at large filling factors is a subject of intense interest. Experimental and theoretical work includes studies of the steady-state response of 2DESs under $a c$ excitation in the microwave (MW) range and under elevated direct current $(\mathrm{dc})$. In both cases, there appears an oscillating magnetoresistance due to scattering-assisted transitions of electrons between different Landau levels (LLs). At low temperatures, when the elastic scattering dominates, such transitions may occur due to coupling of LLs either by the MW field, owing to absorption and emission of the radiation quanta $\hbar \omega$, or by the Hall field $E_{d c}$ which tilts the LLs. Consequently, the microwave-induced resistance oscillations ${ }^{1-4}$ (MIROs) are governed by the ratio $\epsilon_{a c}=\omega / \omega_{c}$ where $\omega_{c}=e B / m$ is the cyclotron frequency, $B$ is the magnetic field strength, and $m$ is the effective mass of electrons. Next, the Hall fieldinduced resistance oscillations ${ }^{5-7}$ (HIROs) are governed by the ratio $\epsilon_{d c}=2 R_{c} e E_{d c} / \hbar \omega_{c}$, where $R_{c}=v_{F} / \omega_{c}$ is the classical cyclotron radius and $v_{F}$ is the Fermi velocity. The Hall field is defined as $E_{d c}=\rho_{H} j$, where $\rho_{H}=m \omega_{c} / e^{2} n_{s}$ is the Hall resistivity, $n_{s}$ is the electron density, and $j=I_{d c} / w$ is the current density with $I_{d c}$ being the applied current and $w$ the sample width.

The amplitude of MIROs can increase dramatically with increasing MW power. In the samples with high electron mobility, elevated MW power transforms the minima of these oscillations into intervals of zero dissipative resistance. These are referred to as zero-resistance states (ZRSs). ${ }^{2-4}$ The amplitude of HIROs is considerably smaller because these oscillations rely upon the large-angle scattering between field-tilted LLs, which requires the presence of short-range scattering potential. However, in the regime $\epsilon_{d c}<1$, corresponding to the first minimum of the HIROs, the Hall field creates a strongly nonequilibrium electron distribution within the LLs, which causes a considerable decrease of the resistance. ${ }^{8}$ This may give rise to a phenomenon of vanishing differential resistance in high-mobility $2 \mathrm{DESs} ;{ }^{9}$ the resulting states are called zero-differential-resistance states (ZDRSs). The ZDRSs can also emerge from the Shubnikov-de Haas $(\mathrm{SdH})$ oscillation maxima. ${ }^{10}$ The ZDRS phenomenon in the $d c$ response resembles the ZRSs in the MW-induced response and can be explained with similar reasons, in terms of the negative differential resistivity, which leads to an instability of the homogeneous current picture and spontaneous breaking of the sample into current domains. ${ }^{11}$ As the instability is of macroscopic origin, it exists regardless of the details of the microscopic mechanisms of nonequilibrium magnetoresistance. However, these mechanisms determine the region of the magnetic fields where the ZRSs or ZDRSs are observed.

Furthermore, an interesting situation takes place when a 2DES is subjected to both $d c$ and $a c$ excitations so that both sources of nonlinearity are applied together. Experimental studies ${ }^{12,13}$ have shown that the resulting oscillations depend on $\epsilon_{a c}$ and $\epsilon_{d c}$, as well as on the combination of these parameters, $\epsilon_{a c} \pm \epsilon_{d c}$. The component of the magnetoresistance oscillating with $\epsilon_{a c} \pm \epsilon_{d c}$ can be described as an interference between MIROs and HIROs. A continuous increase of the current transforms the oscillation maxima into minima (and vice versa) and may lead to destruction of ZRSs and to reappearance of ZRSs in a different region of magnetic fields.

A unified physical picture of the nonequilibrium magnetotransport phenomena described above is currently under development. Earlier theoretical works were focused on the influence of MWs on the probabilities of elastic scattering of electrons in a system of LLs, ${ }^{14-16}$ which contribute to the "displacement" mechanism of MW-induced magnetoresistance. Later, the influence of both MWs and $d c$ excitation on the electron distribution function was found to be very important. ${ }^{17}$ The deviation of this function from equilibrium is proportional to the inelastic electron-electron scattering time $\tau_{i n}$, which rapidly increases with decreasing temperature. Thus, the corresponding "inelastic" mechanism of nonlinear magnetoresistance proves to be the most important one in the description of MW-induced magnetoresistance at low 
temperatures, which is also confirmed experimentally. ${ }^{18} \mathrm{~A}$ comprehensive consideration of possible mechanisms of MWinduced magnetoresistance and of their interplay at elevated radiation power and for different kinds of elastic disorder potentials has been presented in Refs. 19 and 20. A theory of $d c$ magnetoresistance comprising both displacement and inelastic mechanisms and describing the response in a wide interval of applied currents (including the region of HIROs) has also been developed. ${ }^{21}$ The interplay of both $d c$ and $a c$ excitations is studied in Refs. 22 and 23, and the theoretical results confirm the basic properties of the oscillating magnetoresistance investigated in the experiments. ${ }^{12,13}$

The nonequilibrium magnetotransport phenomena acquire qualitatively distinct features in systems with two populated electronic subbands, because of the possibility of electron transitions between the two sets of Landau levels associated with these subbands. As a result, even in the linear regime, such systems show magnetointersubband (MIS) oscillations governed by the ratio $\Delta / \hbar \omega_{c}{ }^{24}$ The subband separation energy $\Delta$ can be a few $\mathrm{meV}$ in double quantum wells so that the MIS oscillation period is comparable to the periods of MIROs and HIROs. Under MW excitation, the oscillating magnetoresistance is determined by interference of MIROs and MIS oscillations. ${ }^{25,26}$ A theory based on consideration of the inelastic mechanism of magnetoresistance in a two-subband system satisfactorily explains the observed oscillating resistivity as well as its temperature dependence in the low-temperature region. ${ }^{25,27-29}$ Similar to single-subband systems, high-mobility two-subband systems demonstrate the ZRS phenomenon. ${ }^{28}$ The nonlinear response to moderate $d c$ excitation $^{30,31}$ shows a flip (inversion) of MIS oscillations. This behavior signifies an inversion of the quantum contribution to resistivity as a consequence of nonequilibrium electron distribution, in agreement with the theory of Ref. 17. The dependence of the characteristic magnetic field (where the flip occurs) on the current density is in quantitative agreement with the theory. ${ }^{31}$ The response to elevated $d c$ excitation $^{32}$ shows the interference of MIS oscillations with HIROs. Finally, it was recently found ${ }^{33}$ that high-mobility two-subband systems develop several regions of ZDRSs around the peaks of inverted MIS oscillations.

In spite of those recent advances in studying magnetotransport in two-subband systems, some problems are still poorly investigated. In particular, neither a systematic experimental study of HIROs nor their theoretical description has been presented. Preliminary studies ${ }^{33}$ of ZDRSs are limited to low temperatures and low $d c$ currents. The behavior of twosubband systems under the combined action of $d c$ and $a c$ excitations has not been studied. In the present work, we intend to fill these gaps. We undertake studies of nonequilibrium magnetotransport in a high-mobility bilayer electron system formed by a wide quantum well (WQW). The high sample quality enables us to carry out a systematic experimental investigation of $d c$ transport and to describe both the ZDRSs and HIROs which appear at elevated currents. We also show and discuss the influence of a $d c$ electric field on MW-induced ZRSs at a chosen frequency of $143 \mathrm{GHz}$. A supporting theoretical consideration, based upon a generalization of existing theories to the two-subband case, reasonably explains the different regimes of nonlinear magnetotransport.

The paper is organized as follows. Section II briefly presents experimental details. In Sec. III we describe the magnetoresistance measured in a wide range of applied $d c$ excitation and compare our data in the regime of HIROs with the theoretical calculations. In Sec. IV we analyze the influence of moderate and strong $d c$ excitations on the magnetoresistance under MW irradiation. Concluding remarks are given in the last section.

\section{EXPERIMENTAL SETUP}

For our experiments, we use high-quality WQWs with a well width of $45 \mathrm{~nm}$, high electron density $n_{s} \simeq 9.2 \times$ $10^{11} \mathrm{~cm}^{-2}$, and a mobility of $\mu \simeq 1.9 \times 10^{6} \mathrm{~cm}^{2} / \mathrm{V} \mathrm{s}$ after a brief illumination with a red light-emitting diode. Several samples in Hall bar geometry (length $l \times$ width $w$ ) of $(500 \times 200) \mu \mathrm{m}^{2}$ have been studied. The electrons in our system occupy the two lowest subbands with a subband separation of $\Delta=1.4 \mathrm{meV}$, extracted from the periodicity of low-field MIS oscillations. Measurements have been performed between 1.4 and $4.2 \mathrm{~K}$ in a cryostat with a variable-temperature insert. For experiments under MW irradiation, a waveguide is employed to deliver linearly polarized MW radiation down to the sample. We record longitudinal resistance using a current of $1 \mu \mathrm{A}$ at a frequency of $13 \mathrm{~Hz}$ in the linear regime. Direct current $I_{d c}$ was applied simultaneously through the same current leads to measure the differential resistance $r_{x x} \equiv$ $d V_{x x} / d I_{d c}$. In the next section we describe the measurements of the resistance under $d c$ excitation and its theoretical analysis.

\section{MAGNETORESISTANCE UNDER dc EXCITATION}

In Fig. 1 we present the differential resistance $r_{x x}$ as a function of the magnetic field for $I_{d c}=0,50 \mu \mathrm{A}$, and $150 \mu \mathrm{A}$.

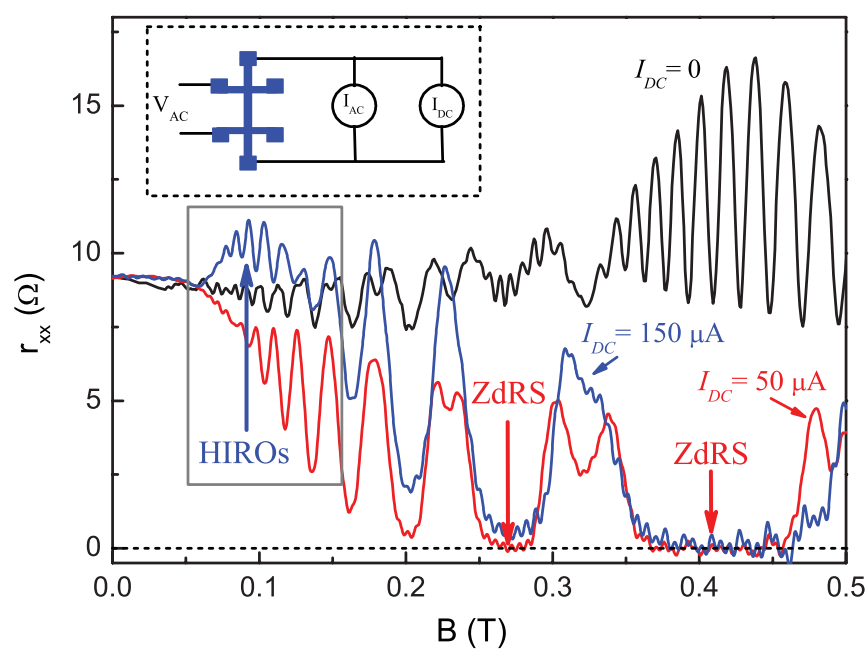

FIG. 1. (Color online) Differential resistance $r_{x x}$ as a function of the magnetic field at $1.4 \mathrm{~K}$. ZDRSs are present for high dc current (arrows). In addition, HIROs appear for $I_{d c}=150 \mu \mathrm{A}$ (see the box) at low magnetic fields. Inset: measurement setup for differential resistance. 

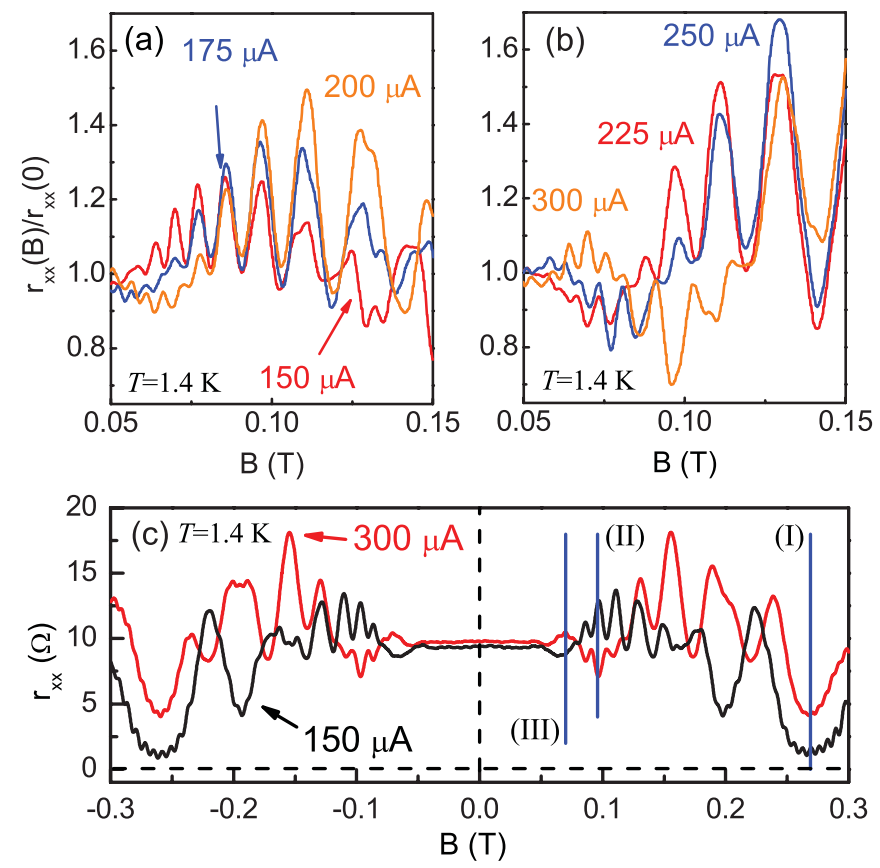

FIG. 2. (Color online) (a), (b) Normalized differential resistance $r_{x x}(B) / r_{x x}(0)$ as a function of the magnetic field for several chosen dc currents showing HIROs for elevated $I_{d c}$. (c) Differential resistance $r_{x x}$ for 150 and $300 \mu \mathrm{A}$. Several MIS oscillations (fixed $B$ ) are marked with (blue) lines and named from I to III. No $\mathrm{SdH}$ oscillations are present in the chosen range of magnetic fields.

For $I_{d c}=0$, the magnetoresistance exhibits well-developed MIS oscillations and thereby confirms the existence of two populated subbands. ${ }^{24}$ It should be noticed that the $a c$ current of $1 \mu \mathrm{A}$ is already high enough to invert MIS oscillations for $B<0.2 \mathrm{~T}$. At $1.4 \mathrm{~K}$, we also observe $\mathrm{SdH}$ oscillations which are superimposed on the MIS oscillations for $B>0.25 \mathrm{~T}$. We first apply a moderate current of $50 \mu \mathrm{A}$ and find that the MIS oscillations are inverted in the whole range of magnetic fields. Around 0.27 and $0.4 \mathrm{~T}$, the inverted MIS peaks evolve into wide regions of ZDRSs. We also find a splitting of the oscillation maxima, e.g., at 0.23 and $0.32 \mathrm{~T}$, similar to that in dc-driven nonlinear magnetotransport in double quantum wells at a high current. ${ }^{31}$ A further increase in the $d c$ electric field (see the trace for $I_{d c}=150 \mu \mathrm{A}$ ) leads to vanishing ZDRSs at $B=$ $0.27 \mathrm{~T}$ and to disappearance of the splitting. Apart from that, the most intriguing features appear around $B=0.1 \mathrm{~T}$, where the inverted MIS peaks are transformed into enhanced ones. Other MIS peaks, e.g., at $0.2 \mathrm{~T}$, remain inverted. We identify the feature around $0.1 \mathrm{~T}$ with the first HIRO peak. Indeed, if $I_{d c}=150 \mu \mathrm{A}$ the condition $\epsilon_{d c}=1$, which corresponds to the maximum of the first HIRO peak, occurs at $B \simeq 0.07 \mathrm{~T}$, not far from the point $B \simeq 0.09 \mathrm{~T}$ where we actually observe the maximum. The shift of the observed maximum to higher fields occurs because the oscillating contribution to resistivity is exponentially suppressed by the disorder in the region of low magnetic fields.

We now focus on HIROs at elevated currents. Figures 2(a) and 2(b) give an overview of magnetoresistance under $d c$ excitation. In this range of magnetic field from 0.05 to $0.15 \mathrm{~T}$
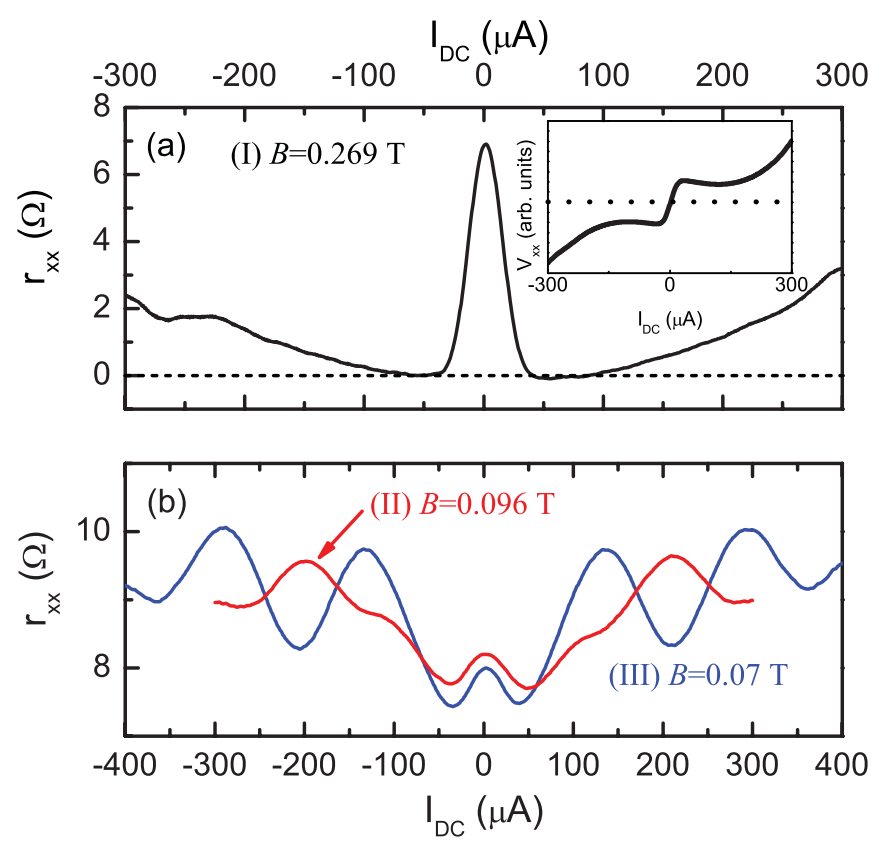

FIG. 3. (Color online) Differential resistance as a function of the applied dc current $I_{d c}$ for the inverted MIS peak which develops into a ZDRS (a) and for two chosen MIS peaks II $(B=0.096 \mathrm{~T})$ and III ( $B=0.07 \mathrm{~T}$ from -400 to $400 \mu \mathrm{A}$ ) at lower magnetic fields (b).

and at a temperature of $1.4 \mathrm{~K}$, MIS oscillations are strongly influenced by a $d c$ electric field. The SdH oscillations, which occur at higher magnetic fields, are damped due to electron heating. For $I_{d c}$ between 150 and $200 \mu \mathrm{A}$, we first see that all MIS oscillations that were inverted for $50 \mu \mathrm{A}$ are now strongly enhanced at $B>0.07 \mathrm{~T}$. In addition, the features around $0.06 \mathrm{~T}$ in Fig. 2(a) become inverted once again with increasing $d c$ excitation. A further increase in the $d c$ excitation from 225 to $300 \mu \mathrm{A}$ modifies the differential magnetoresistance in a similar way, by modulating the MIS oscillations according to the HIRO periodicity. This behavior is also illustrated in Fig. 2(c), where we plot $r_{x x}$ for both negative and positive $B$. In order to study MIS oscillation behavior with increasing electric field, we mark three MIS peaks I to III, which we investigate in detail in Fig. 3.

In Fig. 3(a) we first show $r_{x x}$ as a function of $I_{d c}$ at $B=$ $0.269 \mathrm{~T}$ (as well as the corresponding voltage $V_{x x}$ ) where we observe a ZDRS. This state is created at $I_{d c}^{\mathrm{min}} \simeq 40 \mu \mathrm{A}$ and is observed until a maximal current $I_{d c}^{\max } \simeq 80 \mu \mathrm{A}$. Figure 3(b) presents the differential resistance as a function of $I_{d c}$ for MIS peaks II and III with the corresponding magnetic fields indicated. For $0.07 \mathrm{~T}$ (III), the resistivity shows oscillations which are clearly periodic with the current, and the period corresponds to that of HIROs.

In Fig. 4 we show the normalized differential resistance as a function of $B$ at a temperature of $4.2 \mathrm{~K}$. The behavior is basically the same as in Fig. 2. For $I_{d c}=0$, we observe MIS oscillations whereas $\mathrm{SdH}$ oscillations are not present anymore. The amplitudes of HIROs are weakened at higher temperatures due to enhancement of inelastic electron-electron scattering and the corresponding decrease of the quantum lifetime of electrons. $^{20,34}$ 

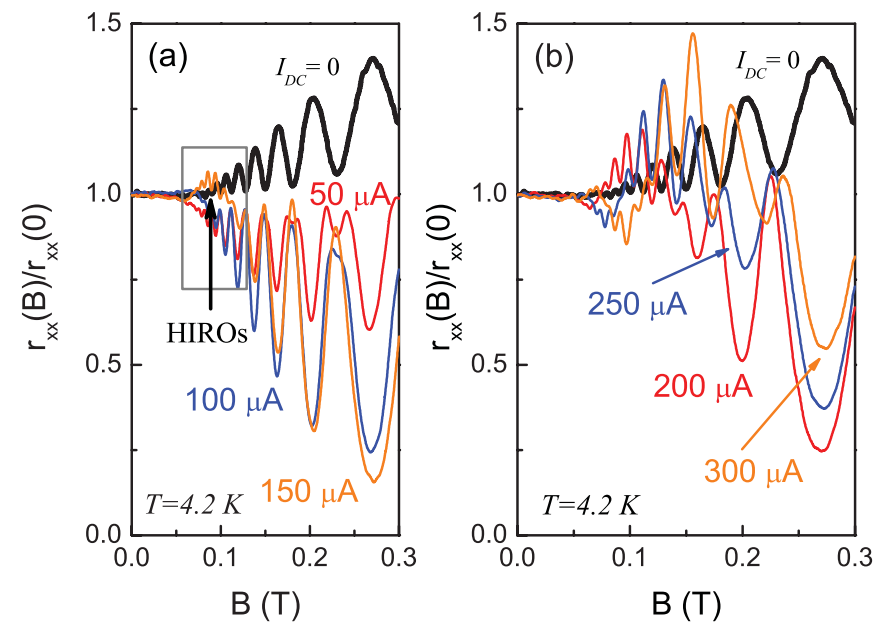

FIG. 4. (Color online) HIROs at a temperature of $4.2 \mathrm{~K}$ (a) for $I_{d c}=0,50,100$, and $150 \mu \mathrm{A}$ and (b) for 200, 250, and $300 \mu \mathrm{A}$. The oscillating response to dc excitation weakens with increasing temperature. $\mathrm{SdH}$ oscillations are not present in the chosen range of magnetic field at this temperature.

Let us analyze the observed behavior in more detail. A theory which satisfactorily describes the origin of ZDRSs in our samples has been presented in Ref. 33. For the regions of magnetic field around 0.27 and $0.4 \mathrm{~T}$, corresponding to inverted MIS peaks, the differential resistivity calculated within the assumption of homogeneous current appears to be negative if $T$ is low enough. The negative differential resistivity, according to Ref. 11, leads to current domains and to ZDRSs in the observed magnetoresistance, as in the case of single-subband systems. ${ }^{9,10}$ The theory ${ }^{33}$ also explains the splitting of the maxima between inverted MIS peaks as a result of the influence of Landau quantization on inelastic electron-electron scattering in two-subband systems (see also Ref. 31). The effects of higher currents described above are consistent with this theory. In particular, the ZDRS is suppressed owing to heating of the 2DES and the related decrease of the quantum lifetime of electrons, which leads to a suppression of all kinds of magnetoresistance oscillations, especially for lower magnetic fields. The splitting disappears because at elevated currents $\left(\epsilon_{d c} \sim 1\right)$ the influence of electron-electron scattering on the distribution function of electrons becomes inessential, ${ }^{21}$ this function is stabilized by the Hall field rather than by the inelastic scattering. We have confirmed these properties by a direct numerical calculation of the magnetoresistance. By including the short-range scattering potential into the theory (see the details below), we also checked the presence of the first HIRO peak near $B=0.1 \mathrm{~T}$ at $I_{d c}=150 \mu \mathrm{A}$. This peak coexists with the ZDRS around $0.4 \mathrm{~T}$, in full accordance with our experimental data (see Fig. 1).

We now concentrate on theoretical calculations of magnetoresistance under strong $d c$ excitation and weak magnetic fields, in the regime where HIROs are observed. In the description of electron transport, we assume elastic scattering of electrons by a random static potential of impurities or other inhomogeneities. If the region of magnetic field below $0.3 \mathrm{~T}$ we apply the approximation of overlapping Landau levels. The quantum contribution to resistivity in this regime is propor- tional to the square of the Dingle factor $d=\exp \left(-\pi / \tau_{q} \omega_{c}\right)$, where $\tau_{q}$ is the quantum lifetime of electrons. Under these conditions, nonlinear magnetoresistance in single-subband systems is described by the theory of Ref. 21. A generalization of this theory to two-subband systems is straightforward since the subband separation $\Delta$ is small compared to the Fermi energy $\varepsilon_{F}$, so the difference in Fermi velocities and scattering rates for different subbands can be neglected. In the regime of classically strong magnetic fields, the resistivity of the electron system is found from the following relation between the density of the applied current $j=I_{d c} / w$ and the longitudinal electric field $E_{\|}=V_{x x} / l$ :

$$
\begin{aligned}
E_{\|}= & j \rho_{0}\left\{1-d^{2}\left(1+\cos \frac{2 \pi \Delta}{\hbar \omega_{c}}\right)\right. \\
& \left.\times\left[\frac{\partial^{2} \gamma(\zeta)}{\partial \zeta^{2}}+\frac{2(\partial \gamma(\zeta) / \partial \zeta)^{2}}{\tau_{t r} / \tau_{i n}+\tau_{t r} / \tau_{q}-\gamma(\zeta)}\right]\right\},
\end{aligned}
$$

where $\rho_{0}=m / e^{2} n_{s} \tau_{t r}$ is the classical Drude resistivity, $\tau_{t r}$ is the transport time, and $\tau_{\text {in }}$ is the inelastic scattering time. The dimensionless function $\gamma(\zeta)$ is given by the expression

$$
\gamma(\zeta)=\int_{0}^{2 \pi} \frac{d \theta}{2 \pi} J_{0}[2 \zeta \sin (\theta / 2)] \frac{\tau_{t r}}{\tau(\theta)}=\sum_{k} \frac{\tau_{t r}}{\tau_{k}} J_{k}^{2}(\zeta),
$$

where $J_{k}(x)$ are the Bessel functions and $1 / \tau(\theta)$ is the angulardependent scattering rate at the Fermi energy. The integral in Eq. (2) describes the averaging over the scattering angle $\theta$. The second part of Eq. (2) shows an equivalent representation of $\gamma(\zeta)$ in terms of angular harmonics of this scattering rate. ${ }^{21}$ The transport time $\tau_{t r}$ is defined in the usual way, as the inverse of the angular averaging of the factor $(1-\cos \theta) / \tau(\theta)$. The dimensionless parameter $\zeta=\pi \epsilon_{d c}$ characterizes the Hallfield-induced tilt of Landau levels. One can write $\zeta$ through the current density as

$$
\zeta=\sqrt{\frac{4 \pi^{3} j^{2}}{e^{2} n_{s} \omega_{c}^{2}}} .
$$

The formal difference of the theory described by Eqs. (1)-(3) from the single-subband theory of Ref. 21 is given by the MIS oscillation factor $\left[1+\cos \left(2 \pi \Delta / \hbar \omega_{c}\right)\right] / 2$ in the quantum contribution to the resistivity and by an extra factor of $1 / \sqrt{2}$ in Eq. (3).

The calculation of the differential resistance from Eqs. (1)-(3) is based upon the model of mixed disorder. ${ }^{20-22}$ We assume that the random static potential contains two components: the short-range and the long-range ones. The corresponding scattering rate is expressed as

$$
\frac{1}{\tau(\theta)}=\frac{1}{\tau_{s}}+\frac{1}{\tau_{l}(\theta)}
$$

where the angle-independent $\tau_{s}$ gives the contribution of the short-range potential to the total scattering rate. The longrange contribution is approximated by an exponential form $\tau_{l}^{-1} \propto \exp \left(-l_{c} q\right)$, where $\hbar q=2 p_{F} \sin (\theta / 2)$ is the transferred momentum, $p_{F}$ is the Fermi momentum, and $l_{c}$ is the correlation length of the potential. For numerical calculations, we have used $\tau_{t r}$ found from the zero-field mobility and determined the quantum lifetime at $T=4.2 \mathrm{~K}$ as $\tau_{q}=6 \mathrm{ps}$, based on the comparison between the measured and calculated 

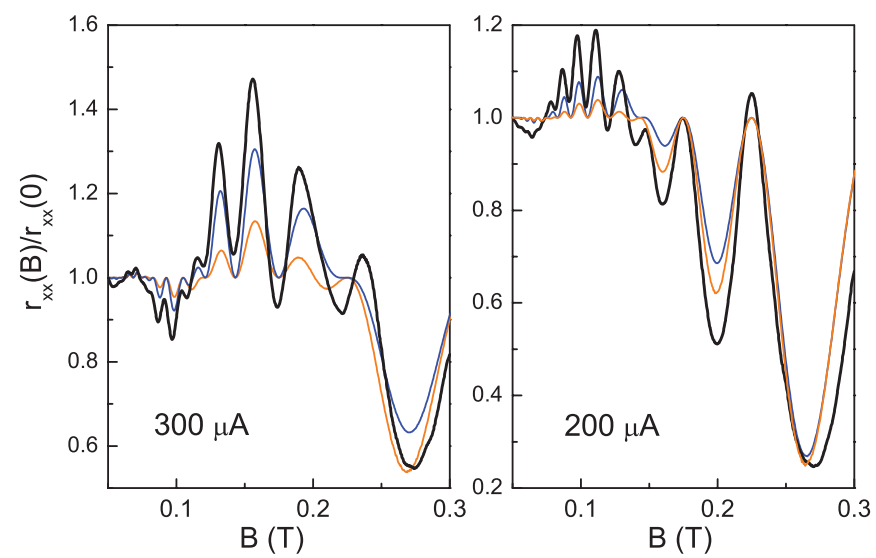

FIG. 5. (Color online) Normalized differential resistance at $T=4.2 \mathrm{~K}$ for $I_{d c}=300$ and $200 \mu \mathrm{A}$. Broad lines (black) are the experimental plots while narrow lines show the calculated magnetoresistance for the cases when the relative contribution of short-range scatterers to the transport scattering rate $1 / \tau_{t r}$ is $50 \%$ and $90 \%$. The amplitudes of the oscillations increase with increasing content of the short-range scatterers.

magnetoresistance in the linear transport regime. The inelastic scattering time is estimated ${ }^{17}$ as $\tau_{i n} \simeq \hbar \varepsilon_{F} / T_{e}^{2}$, where $T_{e}$ is the electron temperature. The appreciable heating of the electron gas by the applied field is obtained from the energy balance equation, taking into account the energy loss due to electron-phonon interaction (for example, a current of $300 \mu \mathrm{A}$ is found to heat electrons from $T=4.2 \mathrm{~K}$ to $T_{e} \simeq 4.8 \mathrm{~K}$ ). In Fig. 5 we plot the calculated normalized magnetoresistance for two different values of the applied current $I_{d c}$ and compare it with the experimental magnetoresistance. While for both currents we have a good agreement in the HIRO periodicity, the amplitudes of the oscillations expected from the theory are smaller than the experimental ones in the low-field part of the plot. Naturally, since the HIROs exist due to backscattering processes, their amplitudes increase with increasing content of the short-range scatterers. This also leads to a better agreement between the theory and the experiment. Therefore, based on the large HIRO amplitudes observed, we may conclude that the amount of short-range scatterers in our samples is significant.

\section{INFLUENCE OF dc EXCITATION ON THE MW-INDUCED MAGNETORESISTANCE}

In this section, we present experimental results for the combined action of $d c$ and $a c$ excitations in our bilayer system. As shown in Ref. 28, a ZRS occurs around $B=0.27 \mathrm{~T}$ for $143 \mathrm{GHz}$ at a temperature of $1.4 \mathrm{~K}$. We focus on that particular frequency with the corresponding MW electric field of $E_{\omega} \simeq$ $4.2 \mathrm{~V} / \mathrm{cm}$ for $0 \mathrm{~dB}$ attenuation. As in previous studies, ${ }^{25,27,28}$ MIROs and ZRSs are not sensitive to the orientation of linear polarization. The immunity of MIROs and ZRSs to the sense of circular polarization has also been found in Ref. 35 where the authors employed a quasioptical setup in which linear and any circular polarization can be produced in situ.

In Fig. 6 we plot the differential magnetoresistance for $I_{d c}=0$ and for different $d c$ excitations. The zero-bias $\left(I_{d c}=\right.$ 0 ) trace shows a ZRS developed from the inverted MIS peak

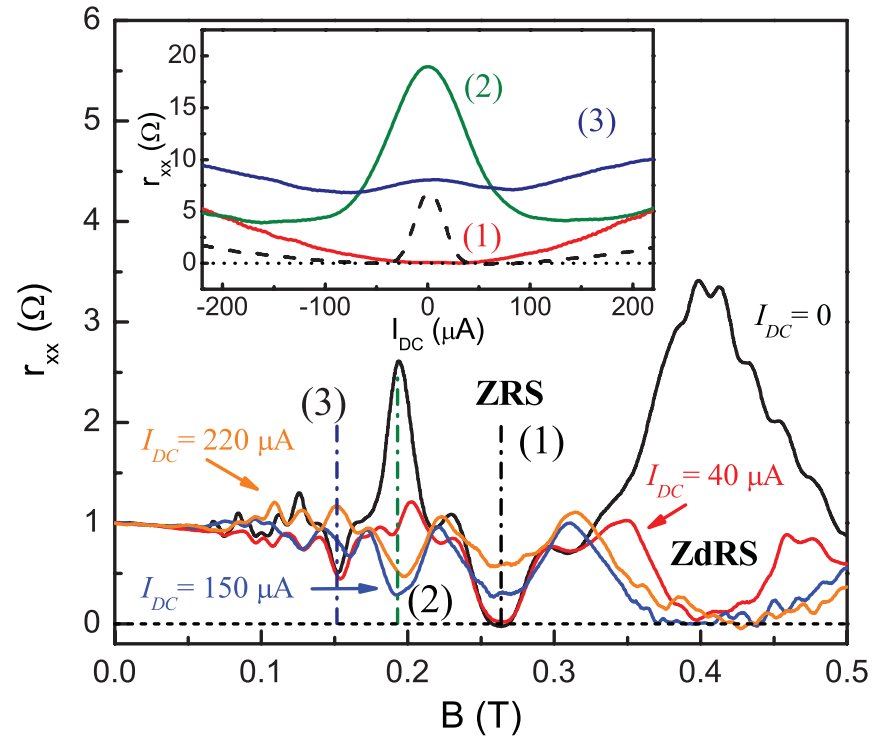

FIG. 6. (Color online) Magnetoresistance under an $a c$ excitation of $143 \mathrm{GHz}\left(I_{d c}=0\right)$ and under combined ac and dc for several chosen $d c$ currents of $I_{d c}=40,150$, and $220 \mu \mathrm{A}$. The ZRS at $0.27 \mathrm{~T}$ is destroyed for 150 and $220 \mu \mathrm{A}$. A ZDRS appears under MW excitation from the enhanced MIS oscillation at $B=0.4 \mathrm{~T}$ for $I_{d c}>40 \mu \mathrm{A}$. Inset: $r_{x x}$ under MW excitation for fixed $B$ for MIS peaks from 1 to 3 as a function of $I_{d c}$ [dashed line without MWs; see also Fig. 3(a)].

at $B=0.27 \mathrm{~T}$ (dash-dotted line 1), the other inverted MIS peaks at 0.15 (line 3 ) and $0.1 \mathrm{~T}$, as well as strongly enhanced MIS peaks around $0.19 \mathrm{~T}$ (line 2) and $0.4 \mathrm{~T}$. If we apply a $d c$ bias starting with $I_{d c}=40 \mu \mathrm{A}$, we obtain a strongly modified oscillation pattern: the MW-enhanced MIS peaks exhibit strong damping or even inversion, while the effect of $I_{d c}$ on the MW-inverted MIS peaks (including the one transformed into a ZRS) is very weak. A further increase in $d c$ excitation has two consequences: (i) the ZRS around $B=0.27 \mathrm{~T}$ vanishes and (ii) a ZDRS appears under MW irradiation in a wide region around $B=0.4$ T. For the highest $d c$ excitation of $I_{d c}=220 \mu \mathrm{A}$, we also find well-developed HIROs for $B<0.2 \mathrm{~T}$. The dependence of the magnetoresistance on the $d c$ current is nonmonotonic for the traces 2 and 3 . We have also carried out $B$ sweeps at different $I_{d c}$ up to $220 \mu \mathrm{A}$ (not shown here) and measurements of the resistance at constant magnetic field as a function of $I_{d c}$ (see inset of Fig. 6). For the ZRS at $B=0.27 \mathrm{~T}$, we find that a $d c$ excitation leads to a vanishing ZRS for $I_{d c} \geqslant 50 \mu \mathrm{A}$, which corresponds to an electric field $E_{d c}>37 \mathrm{~V} / \mathrm{m}$. At $B=0.41 \mathrm{~T}$, a ZDRS develops under $d c$ excitation for relatively low $d c$ currents, $I_{d c} \simeq 28 \mu \mathrm{A}$ giving rise to $E_{d c} \simeq 39 \mathrm{~V} / \mathrm{m}$.

A remarkable fact is that we see the ZDRS in the same region of fields, $B \simeq 0.27 \mathrm{~T}$, regardless of the presence or absence of $a c$ excitation, although with $a c$ excitation we see the ZDRS even in the linear response. As seen from the inset to Fig. 6, this state vanishes for $I_{d c} \geqslant 50 \mu \mathrm{A}$. For comparison, in the case of pure $d c$ excitation the ZDRS appears at $I_{d c} \geqslant 40 \mu \mathrm{A}$ and vanishes at $I_{d c} \geqslant 80 \mu \mathrm{A}$; see also Fig. 3(a). The observed behavior is imposed by the ability of both kinds of excitation to create in this region of magnetic fields a partially inverted electron energy distribution which leads to negative resistance. 
Thus, our observation is consistent with the domain model for the origin of both ZRSs and ZDRSs.

The basic features of the observed behavior can be understood in terms of interplay of the effects of $a c$ and $d c$ excitations. The MW excitation either enhances or inverts MIS peaks (or groups of such peaks) in accordance with MIRO periodicity: ${ }^{25}$ the enhancement occurs around $\epsilon_{a c}=n+3 / 4$ (MIRO maxima) while the inversion corresponds to $\epsilon_{a c}=$ $n+1 / 4$ (MIRO minima), $n$ being an integer. The $d c$ excitation tends to invert all of the MIS peaks in the region of higher magnetic fields, where $\epsilon_{d c}<1$. Thus, the MW-enhanced MIS peaks should be suppressed and eventually inverted by the $d c$ excitation, while the peaks which are already inverted by MWs are expected to be little affected. However, the rapid suppression and inversion of the MW-enhanced MIS peaks already at a moderate $d c$ excitation suggests that the interplay of $a c$ and $d c$ excitations is not reduced to a pure superposition of their effects. To make this point clear, we apply a simple theory valid at $\zeta^{2} \ll 1$ and low temperatures, when the inelastic mechanism of nonlinear response dominates, and use the approximation of overlapping LLs. The nonlinear conductivity of a single-subband system in this case is given by Eq. (15) of Ref. 17 and can be straightforwardly generalized for a system with two closely spaced subbands. Hence, the differential magnetoresistance in written in the form

$$
\begin{gathered}
\frac{r_{x x}(B)}{r_{x x}(0)}=1+d^{2}\left(1+\cos \frac{2 \pi \Delta}{\hbar \omega_{c}}\right) F\left(\epsilon_{a c}, \epsilon_{d c}\right), \\
F=\frac{(1+D)^{2}-10(1+D) Q-3 Q^{2}+A(Q-1-D)}{(1+D+Q)^{2}},
\end{gathered}
$$

where $A=2 \pi \epsilon_{a c} \mathcal{P}_{\omega} \sin 2 \pi \epsilon_{a c}, \quad D=\mathcal{P}_{\omega} \sin ^{2} \pi \epsilon_{a c}, \quad \mathcal{P}_{\omega} \propto$ $\tau_{i n} E_{\omega}^{2}$ is a dimensionless quantity proportional to $\mathrm{MW}$ power, ${ }^{17}$ and $Q=\left(\tau_{i n} / 2 \tau_{t r}\right)\left(\pi \epsilon_{d c}\right)^{2}$ is a dimensionless quantity proportional to $I_{d c}^{2}$. In the MIRO minima and maxima, $A= \pm 2 \pi \epsilon_{a c} \mathcal{P}_{\omega}$, respectively, while $D=\mathcal{P}_{\omega} / 2$ in both cases (notice that $|A| \gg D$ for the relevant region $\omega>\omega_{c}$ ). For negative $A$ (MIRO maxima, enhanced MIS peaks), when all the $Q$-dependent terms in the numerator of $F$ have the same sign, the decrease of $r_{x x}$ with increasing $I_{d c}$ goes much faster than in the absence of MWs. Physically, this is related to the stronger effect of the $d c$ field on the electron distribution if the latter is already modified by MWs. This explains the dramatic suppression of the magnetoresistance peaks by the $d c$ excitation. For positive $A$ (MIRO minima, inverted MIS peaks), the MW term $(\propto A)$ in $F$ considerably slows down the effect of $I_{d c}$ on the magnetoresistance: the electron distribution is modified by MWs in such a way that it is only slightly affected by the $d c$ field. However, in both cases the increase in $I_{d c}$ leads to an additional heating of the $2 \mathrm{DES}$. As a result, both $\tau_{i n}$ and $\tau_{q}$ decrease, and so does the amplitude of oscillations. In Fig. 6 we see this effect not only in the vanishing ZRS at $0.27 \mathrm{~T}$ (line 1) but also in decreasing of the amplitude of the inverted peak at $0.19 \mathrm{~T}$ (line 2). We have estimated that the current $I_{d c}=150 \mu$ A leads to an additional heating of the 2DES in our sample by approximately $1 \mathrm{~K}$, which may explain the observed behavior. Below 0.2 T, Eq. (5) is no longer valid for the highest $I_{d c}$ used. In this region of fields, the nonlinear magnetoresistance cannot be simply described by the $a c$ and $d c$ response of the $2 \mathrm{D}$ system (superposition) but rather as an interplay (interference) of MIROs and HIROs.

\section{CONCLUSION}

We have studied magnetotransport in a high-quality bilayer electron system under a strong $d c$ excitation. We have found well-developed HIROs which modulate the MIS oscillations in our bilayers. A theoretical calculation of the nonlinear magnetoresistance gives a good agreement with experiment concerning the shape and periodicity of the oscillation picture. The comparison of theory and experiment indicates a significant role of electron scattering by the short-range random potential in our samples. Furthermore, we show that HIROs in the region of low magnetic fields coexist with zero differential resistance at higher fields.

We have also examined nonlinear transport under both $a c$ (microwave) and $d c$ excitations. Even a moderate $d c$ excitation strongly modifies the MW-induced resistance. This effect is consistent with a theoretical model predicting that the combined action of two kinds of excitation is not merely a superposition of $a c$ and $d c$ effects. It is found that for the chosen MW frequency both these excitations lead to ZDRSs in the same region of fields, around $B=0.27 \mathrm{~T}$. The properties of ZDRSs with and without MW excitation are in accordance with the domain model which explains the vanishing resistance as a result of instability of homogeneous current flow under conditions of negative differential resistivity. A better understanding of the interplay of $a c$ and $d c$ excitations is desirable and requires further experimental and theoretical work.

\section{ACKNOWLEDGMENTS}

We acknowledge support from COFECUB-USP (Project No. $\mathrm{U}_{\mathrm{c}}$ Ph 109/08), FAPESP, and CNPq (Brazilian agencies).
${ }^{1}$ M. A. Zudov, R. R. Du, J. A. Simmons, and J. L. Reno, Phys. Rev. B 64, 201311(R) (2001); P. D. Ye et al., Appl. Phys. Lett. 79, 2193 (2001).

${ }^{2}$ R. G. Mani, J. H. Smet, K. von Klitzing, V. Narayanamurti, W. B. Johnson, and V. Umansky, Nature (London) 420, 646 (2002).

${ }^{3}$ M. A. Zudov, R. R. Du, L. N. Pfeiffer, and K. W. West, Phys. Rev. Lett. 90, 046807 (2003).

${ }^{4}$ R. L. Willett, L. N. Pfeiffer, and K. W. West, Phys. Rev. Lett. 93, 026804 (2004).
${ }^{5}$ C. L. Yang, J. Zhang, R. R. Du, J. A. Simmons, and J. L. Reno, Phys. Rev. Lett. 89, 076801 (2002).

${ }^{6}$ A. A. Bykov, J. Q. Zhang, S. Vitkalov, A. K. Kalagin, and A. K. Bakarov, Phys. Rev. B 72, 245307 (2005).

${ }^{7}$ W. Zhang, H.-S. Chiang, M. A. Zudov, L. N. Pfeiffer, and K. W. West, Phys. Rev. B 75, 041304(R) (2007).

${ }^{8}$ J. Q. Zhang, S. Vitkalov, A. A. Bykov, A. K. Kalagin, and A. K. Bakarov, Phys. Rev. B 75, 081305(R) (2007); J. Q. Zhang, S. Vitkalov, and A. A. Bykov, ibid. 80, 045310 (2009). 
${ }^{9}$ A. T. Hatke, H.-S. Chiang, M. A. Zudov, L. N. Pfeiffer, and K. W. West, Phys. Rev. B 82, 041304(R) (2010).

${ }^{10}$ A. A. Bykov, J.-Q. Zhang, S. Vitkalov, A. K. Kalagin, and A. K. Bakarov, Phys. Rev. Lett. 99, 116801 (2007).

${ }^{11}$ A. V. Andreev, I. L. Aleiner, and A. J. Millis, Phys. Rev. Lett. 91, 056803 (2003).

${ }^{12}$ W. Zhang, M. A. Zudov, L. N. Pfeiffer, and K. W. West, Phys. Rev. Lett. 98, 106804 (2007).

${ }^{13}$ A. T. Hatke, H.-S. Chiang, M. A. Zudov, L. N. Pfeiffer, and K. W. West, Phys. Rev. B 77, 201304(R) (2008).

${ }^{14}$ V. I. Ryzhii, Fiz. Tverd. Tela (Leningrad) 11, 2577 (1969) [Sov. Phys. Solid State 11, 2078 (1970)]; V. I. Ryzhii, R. A. Suris, and B. S. Shchamkhalova, Fiz. Tekh. Poluprovodn. 20, 2078 (1986) [Sov. Phys. Semicond. 20, 1299 (1986)].

${ }^{15}$ A. C. Durst, S. Sachdev, N. Read, and S. M. Girvin, Phys. Rev. Lett. 91, 086803 (2003).

${ }^{16}$ M. G. Vavilov and I. L. Aleiner, Phys. Rev. B 69, 035303 (2004).

${ }^{17}$ I. A. Dmitriev, M. G. Vavilov, I. L. Aleiner, A. D. Mirlin, and D. G. Polyakov, Phys. Rev. B 71, 115316 (2005).

${ }^{18}$ A. T. Hatke, M. A. Zudov, L. N. Pfeiffer, and K. W. West, Phys. Rev. Lett. 102, 066804 (2009).

${ }^{19}$ I. A. Dmitriev, A. D. Mirlin, and D. G. Polyakov, Phys. Rev. B 75, 245320 (2007).

${ }^{20}$ I. A. Dmitriev, M. Khodas, A. D. Mirlin, D. G. Polyakov, and M. G. Vavilov, Phys. Rev. B 80, 165327 (2009).
${ }^{21}$ M. G. Vavilov, I. L. Aleiner, and L. I. Glazman, Phys. Rev. B 76, 115331 (2007).

${ }^{22}$ M. Khodas and M. G. Vavilov, Phys. Rev. B 78, 245319 (2008).

${ }^{23}$ X. L. Lei and S. Y. Liu, Appl. Phys. Lett. 93, 082101 (2008).

${ }^{24}$ N. C. Mamani, G. M. Gusev, T. E. Lamas, A. K. Bakarov, and O. E. Raichev, Phys. Rev. B 77, 205327 (2008).

${ }^{25}$ S. Wiedmann, G. M. Gusev, O. E. Raichev, T. E. Lamas, A. K. Bakarov, and J. C. Portal, Phys. Rev. B 78, 121301(R) (2008).

${ }^{26}$ A. A. Bykov, D. P. Islamov, A. V. Goran, and A. I. Toropov, JETP Lett. 87, 477 (2008).

${ }^{27}$ S. Wiedmann, G. M. Gusev, O. E. Raichev, A. K. Bakarov, and J. C. Portal, Phys. Rev. B 81, 085311 (2010).

${ }^{28}$ S. Wiedmann, G. M. Gusev, O. E. Raichev, A. K. Bakarov, and J. C. Portal, Phys. Rev. Lett. 105, 026804 (2010).

${ }^{29}$ J. Iñarrea and G. Platero, Phys. Rev. B 84, 075313 (2011).

${ }^{30}$ A. A. Bykov, JETP Lett. 88, 64 (2008).

${ }^{31}$ N. C. Mamani, G. M. Gusev, O. E. Raichev, T. E. Lamas, and A. K. Bakarov, Phys. Rev. B 80, 075308 (2009).

${ }^{32}$ A. A. Bykov, JETP Lett. 88, 394 (2008); 89, 461 (2009).

${ }^{33}$ G. M. Gusev, S. Wiedmann, O. E. Raichev, A. K. Bakarov, and J. C. Portal, Phys. Rev. B 83, 041306(R) (2011).

${ }^{34}$ A. T. Hatke, M. A. Zudov, L. N. Pfeiffer, and K. W. West, Phys. Rev. B 79, 161308 (2009).

${ }^{35}$ J. H. Smet, B. Gorshunov, C. Jiang, L. Pfeiffer, K. West, V. Umansky, M. Dressel, R. Meisels, F. Kuchar, and K. von Klitzing, Phys. Rev. Lett. 95, 116804 (2005). 